\title{
Energía: Desde un modelo de derroche, hacia un modelo sostenible mediante energía renovable
}

\author{
Juan Alberto Betanco Maradiaga ${ }^{1}$
}

\section{RESUMEN}

Energías renovables se relaciona a la concepción de desarrollo sostenible, que a su vez tiene que ver con los principios de conservar el capital natural, equidad, calidad de vida, paradoja ética, ecoforma, gestión integral, solidaridad inter e intrageneracional, utopía y agenda política global. Así mismo, su conexión con las dimensiones ambientales, demográficas, de consumo, global, local, tecno social y cultural. No se puede entender este paradigma sino se entiende primeramente el modelo capitalista consumista, en que está sumergida la sociedad desde la época de la revolución industrial. Este escrito analiza críticamente el rol de la ciencia, tecnología y energía en el ámbito mundial, vinculado al contexto latinoamericano en general y nicaragüense en particular. Se hace énfasis en las perspectivas de las energías renovables, propiamente en los sistemas a baja escala constituidos por tecnologías hibridas, como la eólico solar fotovoltaica para su uso en zonas rurales y urbanas.

Palabras clave: desarrollo sostenible, principios, energía, ciencia, tecnología, energías renovables.

Recibido: 20 de mayo de 2017

Aceptado: 28 de agosto de 2017

1 Docente titular Universidad Nacional Autónoma de Nicaragua, FAREM-Estelí. Doctorando del programa Gestión y Calidad de la Investigación Científica. FAREM Estelí-UNAN Managua. Correo electrónico: juan.betanco@yahoo.com 


\title{
Energy: From a waste model, towards a sustainable model using renewable energy
}

\begin{abstract}
Renewable energy is related to the conception of sustainable development, which is related to the principles of conserving natural capital, equity, quality of life, ethical paradox, ecoform, integral management, inter and intragenerational solidarity, utopia and global political agenda. Likewise, its connection with the environmental, demographic, consumer, global, local, techno social and cultural dimensions. This paradigm can not be understood unless the capitalist consumerist model is understood first, in which society has been submerged since the time of the industrial revolution. This paper critically analyzes the role of science, technology and energy in the world, linked to the Latin American context in general and Nicaraguan in particular. Emphasis is also placed on the perspectives of renewable energies, properly on the small scale systems constituted by hybrid technologies such as photovoltaic solar wind for use in rural and urban areas.
\end{abstract}

Keywords: sustainable development, principles, energy, science, technology, renewable energies 


\section{INTRODUCCIÓN}

En la complejidad del presente siglo hablar de energía conduce a analizar un enfoque integral que abarca la ciencia, la tecnología, la sociedad, el medio ambiente y desarrollo sostenible. Se torna más complejo por las desigualdades e iniquidades del mundo en que vivimos, que ha creado crisis estructurales y provocado la construcción de esquemas mentales de dependencia, consumismo y falso desarrollo.

La relación del ser humano con la naturaleza ha sido desequilibrada en todos los sentidos. Ha predominado una relación dicotómica del yo y lo demás en provecho del yo, sin considerar que el ser humano es parte de la naturaleza misma. Edgar Morin (2000), estudioso del pensamiento complejo menciona: "somos resultado del cosmos, de la naturaleza, de la vida, pero debido a nuestra humanidad misma, a nuestra cultura, a nuestra mente, a nuestra conciencia, nos hemos vuelto extraños a este cosmos que nos es secretamente íntimo." (pág. 25). Esa visión sobre la naturaleza la ha hecho instrumento del ser humano como una fuente infinita de recursos, alterando drásticamente el planeta hasta convertir la situación en insostenible. Este estilo de vida difundido por el capitalismo, es habitual desde la revolución industrial hasta nuestros días creando la concepción de que desarrollo se refiere a tener dinero, aspectos materiales y formas de vida dictadas por las modas del capital.

La energía eléctrica como bien, no se ha escapado a la manipulación del poder y ha sido factor determinante para las desigualdades entre la riqueza y la pobreza. Hay grandes ciudades súper iluminadas, hoteles en zonas desérticas con arenas refrigeradas, ciudades construidas en el mar, caracterizadas por grandes derroches de energía, mientras en África, América Latina y algunos pueblos de Asia, grandes sectores de la población no tienen acceso a la energía eléctrica y gastan sus ganancias en pago de combustible fósiles que tienen que importar. Aquí cabe la pregunta; ¿Es pertinente cambiar de paradigma?
El paradigma de desarrollo sostenible se ha propuesto transformar esta sociedad de manera profunda rompiendo esquemas mentales y proponer un nuevo tipo de desarrollo que tenga en cuenta una visión interconectada a la realidad en la cual se consideren los aspectos sistémicos y multidimensionales de sistemas físicos, biológicos, mentales y culturales en constante proceso de cambio.

El concepto mismo de desarrollo sostenible es un concepto en evolución, como mencionan Cendra y Stahel (2006), "desde la que se ha convertido en la definición oficial del concepto, formulada por la comisión Brundtland, hasta nuestros días, muchas son las críticas que se le han hecho y muchos los debates establecidos desde sistemas de valores diversos y a menudo encontrados, que exigirán todavía muchos esfuerzos de concreción y acuerdo". Sin embargo, como especifica Jabareen (2006) "hay un sentido general que la sostenibilidad es una cosa buena, pero que todavía requiere definición y elaboración" (pág. 2). Por lo mismo se debe afrontar la realidad social, económica, política, ambiental y cultural de los países latinoamericanos desde una visión común, acorde con la historia de cada país y contribuir a la construcción social del concepto de desarrollo sostenible teniendo en cuenta las interrelaciones de cada país.

El desarrollo sostenible no puede ser réplica del desarrollo en otros países, sino el desarrollo propio creado y concebido por cada persona como parte de la naturaleza y su entorno en cada país, guiado por factores multifacéticosy sistémicodecomportamientocomplejo. Se trata de establecer una nueva forma de relación con lo demás como o menciona Ritter (2017) en su artículo: "Necesitamos de una metodología diferente para tratar con sistemas que son intrínsecamente diferentes hasta lo ahora considerado (...) que interactúan en forma dinámica y de multimaneras, siguiendo reglas locales e independientes de cualquier instrucción de un nivel superior. Mucho más importante que el mero número de variables es el hecho de que estas variables están interrelacionadas". 
En el aspecto energético se trata de identificar el capital natural con potencial energético para su aprovechamiento, evitando su deterioro, en consenso con los actores territoriales sin perjudicar, manteniendo la equidad y la solidaridad inter e intrageneracional. Los países latinoamericanos poseen un enorme capital natural que aprovechado de manera sostenible puede garantizar energía para esta y las futuras generaciones. En ese sentido, Nicaragua viene haciendo esfuerzos por establecer un nuevo modelo de uso de energía que aproveche la riqueza de recursos geotérmicos, hídricos, eólicos, solares y de biomasa que posee para alcanzar el cambio de la matriz energética y el acceso de todos los nicaragüenses a la energía eléctrica.

En la primera parte de este artículo se revisa el concepto de desarrollo sostenible basado en el análisis de sus dimensiones y principios, sus implicaciones y consecuencias concretas en la sociedad nicaragüense con el propósito de contribuir a la concreción científica del concepto de desarrollo sostenible. Este enfoque parte desde el contraste de los paradigmas existentes. En la segunda parte se aborda el papel clave de la ciencia, tecnología y su impacto en la sociedad enfocado desde el aspecto de las energías renovables; sus ventajas y desventajas. La última parte se enfoca al contexto energético actual, mundial y las perspectivas latinoamericanas, en particular de Nicaragua en pro del desarrollo sostenible ligado al aprovechamiento de los recursos energéticos y la tecnología para lograrlo; entre la que se describe la generación de energía eléctrica por sistemas eólicos.

\section{MÉTODO}

El presente escrito se basa en una revisión bibliográfica del modelo de crecimiento actual desde la revolución industrial hasta el presente analizando su impacto en la sociedad. Han servido de fuentes primarias libros, revistas y escritos que han sido seleccionados por sus enfoques de pensamiento complejo, sistémicos y holísticos que describen pormenores del modelo consumista de desarrollo. El análisis de un nuevo paradigma de desarrollo sostenible como menciona Jaume Cendra Garreta (2006), "bajo los criterios de principios y dimensiones del desarrollo sostenible", se realiza desde la ubicación de varias fuentes documentales primarias y secundarias, buscadas en google académicos, bases de datos digitales, repositorios de artículos y archivos de las naciones unidas. En ambos casos se ha destinado tiempo a la lectura crítica de los documentos relacionando las semejanzas o diferencias en las argumentaciones.

Para el aspecto relacionado a la ciencia, tecnología y sociedad se centra la búsqueda de información en documentación relacionada a Latinoamérica con el objetivo de identificar aspectos relevantes conocidos, desconocidos y controvertidos como menciona Osvaldo Sunkel (1969), sobre las reflexiones del "papel de la ciencia y la tecnología en los países en desarrollo en contraste con el mismo papel de las mismas categorías pero en países desarrollados".

La situación energética está basada en datos buscados y seleccionados por su actualización en fuentes de OLADE, CEPAL, balances energéticos con el objetivo de describir el estado de las fuentes renovables en general y en el área de eólica en particular.

\section{RESULTADOS DEL ANALISIS}

\section{Un modelo de desarrollo de capitales basado en la explotación irracional de la energía}

El modelo actual de desarrollo ha introducido a la humanidad entera en el desorden, el caos, en lo insostenible, en la incertidumbre y en la ruina. No se trata tampoco de morir consciente de que todo está acabado y que ya no se puede hacer nada. Al contrario, es hurgar entre lo que queda, nutrirse del caos y la incertidumbre para crear la certidumbre, lo sostenible. Buscar en el atraso para despegar y reconstruir. Es transformar nuestro pensamiento y hasta nuestro conocimiento de la verdad. 
El desarrollo humano se ha caracterizado por el afán de vivir y no de vivir bien. Según Fernández y González (2014), ese afán de vivir por vivir unido a la acumulación de dinero empezó a tener forma con la revolución industrial en el sistema de compra y venta del libre mercado volcada a la producción de excedente, profundizando la desigualdad y la brecha entre ricos y pobres. Lo definió el propio Adam Smith (1776) "Todo hombre es rico o pobre según el grado en que pueda gozar de la cosas necesarias, convenientes y gratas de la vida". De ahí que el vivir se ligue a ganar dinero para gozar y se confunda riqueza con dinero. He aquí el primer desorden encontrado, que excluye de la riqueza la ecología, lo social y la cultura; dejando por fuera el sol, el aire que se respira, las plantas, los animales, el agua, las relaciones familiares y sociales, como asuntos gratos para la vida.

Se ha desarrollado una sociedad consumista, creando dependencia en las personas de productos innecesarios. Las necesidades de rentabilizar las inversiones de capitales en los nuevos productos creados por los grandes monopolios, han generado un continuo estado de insatisfacción individual y la adicción a necesitar y consumir. Así se cae en líneas sin fin de necesidades, de nuevos celulares, computadoras, automóviles, electrodomésticos, adquisición de nuevas medicinas que ocultan los efectos secundarios, alimentos que no tienen que ver con su calidad dietética si no con estilos de vidas. Como señala Fernández y González (2014), se han centrado en "la creación y el fomento del sentido de escasez y, sobre todo, la constante generación de nuevos caprichos y deseos, apelando sobre todo a las emociones".

Todos estos nuevos productos requieren de energía, de materia prima proveniente de los recursos naturales para su fabricación, creando un modelo de consumismo y de desechos que tiene asfixiado al planeta contaminando aguas superficiales y subterráneas, el suelo, el aire. Se han creado monopolios para mayor acumulación de capital desarrollando la industria de armamentos, petróleo, automóviles, procesadores y software que no contribuyen a la riqueza social y ambiental. Aquí se localiza otro caos en el ciclo sin fin del consumismo del disfrute, que estimula el despilfarro, un desorden insostenible en la explotación de los recursos naturales, socavando de esa manera sus propios sustentos y la falta de equidad en la distribución de las ganancias que producen los negocios. Este modelo de desarrollo en vez de reducir las desigualdades sociales ha abonado la pobreza, manifestándose mediante la escasez, cayendo en ese ciclo vicioso de trabajar honradamente, pero vulnerables al consumo desordenado.

La ciencia y la tecnología a lo largo de la historia se han desarrollado, pero no todo es positivo; han servido de soporte al modelo impulsando la compra indiscriminada de nuevas cosas costosas e ineficientes y a la vez han convertido a los países pobres en compradores de equipos en proceso de descarte. Las nuevas tecnologías crean nuevos problemas éticos relacionados al medio ambiente y la informática da una nueva pauta como forma de discriminación y de amenaza a la vida privada. La televisión supone una avalancha de noticias, diversión y glamour, especialmente a través de la publicidad, que logra apartar la atención humana del mundo físico y el internet ha puesto en manos del estado y las grandes trasnacionales un gran control de los seres humanos. El funcionamiento del ciberespacio demanda una considerable cantidad de energía eléctrica. Actividades, como la lectura de un periódico en línea, el envío de gran cantidad de datos vía correo electrónico, el libro electrónico o colgar vídeos, tienen también su costo energético, como señalan Fernández y González (2014).

García y Tejado (2012) al respecto manifiestan; "ni internet, ni las redes sociales ni ninguna herramienta tecnológica nos hará más libres, al igual que no nos ha hecho más iguales, ya que han sido diseñadas para acelerar el consumo, no para alimentar la revolución"; ya que en determinados sectores la revolución digital ha sido un atraso, porque empobrece el pensamiento, el conocimiento y la cultura. La ciencia y la tecnología es un asunto de poder que ha sido dominado por los 
grandes capitales de los países ricos, agrandando la brecha con los países en desarrollo que invierten poco en investigación, desarrollo e innovación.

La tecnología es fundamental para la obtención y apropiación de la energía que tiene implicaciones sociales, políticas, económicas y culturales, ya que se han se han dominado personas y territorios. La crisis energética es irreversible ya que los combustibles fósiles están cercanos a su pico de extracción y no hay energía que pueda sustituir al petróleo. Fernández y González (2014), al respecto mencionan que "el detonante del colapso civilizatorio será el final de los combustibles fósiles baratos y abundantes, pero los elementos que determinarán un nuevo contexto serán el cambio climático y la pérdida de biodiversidad".

En todo esto, la energía ha estado estrechamente vinculada. Todo inicia en la revolución industrial con la tecnología del carbón, que permitió el uso intensivo de la energía fósil y explotar al máximo esta nueva energía, con ventajas sobre la biomasa o leña que era la energía utilizada. Las ventajas del carbón según Fernández y González (2014) son “densidad energética netamente mayor, la tecnología y su poder calorífico permitieron un uso muy versátil; eran abundantes y baratos; son fácilmente almacenables", sobre esta base el capitalismo logro su hegemonía económica y social. Este cambio en la matriz energética transformó el mundo produciendo cantidades enormes de energía que consumieron las nuevas máquinas tanto para su funcionamiento como para su construcción como lo indican Fernández y González (2014), "los desarrollos tecnológicos en general han supuesto un mayor consumo de energía y no un ahorro. Con este derroche se consiguió, básicamente, potencia y capacidad de obtener cantidades mayores de energía".

Los impactos son numerosos. Implica una nueva relación con la naturaleza ya que comenzó la explotación de los recursos naturales y fósiles muy por encima de su recuperación. La sustitución del trabajo humano por las máquinas, obligando a personas al desempleo y permitiendo al empresario bajar los salarios, generando una gran plusvalía para la clase capitalista y otorgándoles mayor poder. Se consiguió obtener mayor cantidad de productos a menor precio, bajando los salarios a los trabajadores que se podían mantener con menos ingresos. Las máquinas podían funcionar las 24 horas, las jornadas laborales se hicieron extensas. La productividad por persona aumentó, la competitividad se incrementó y el mundo fue obligado a sumarse a la lógica del nuevo modo de producción capitalista.

Las personas fueron explotadas en las fábricas de tal manera que a la naturaleza y al ser humano se les explotó brutalmente. Se contamino el aire con metales pesados, el agua, el suelo por el uso excesivo del carbón liberando grandes cantidades de $\mathrm{CO} 2$ a la atmosfera. Se estructuró una fuerte desigualdad entre los que usaban el combustible fósil para los fines industriales y los territorios coloniales.

Con la llegada del petróleo y el motor de combustión interna, la hegemonía de Estados Unidos se consolidó. El petróleo se volvió una cuestión de estado. Junto al petróleo crecieron otras fuentes como el gas natural, el carbón, la energía nuclear y la hidráulica. La biomasa con la leña siguió siendo utilizada por las poblaciones pobres. La electricidad implicó un gran salto en la industria de la comunicación, iluminación la que se convirtió en un nuevo interés de inversión en generación, transporte y distribución de electricidad. A principios del siglo XXI los combustibles fósiles aseguraban un $86 \%$ de las necesidades energéticas mundiales. Según Fernández y González (2014), el petróleo supera al carbón por sus características físicoquímicas del petróleo, que le dotan de versatilidad, concentración energética, facilidad y seguridad de transporte y un fácil almacenaje, además es fácil de extraer y se puede obtener sub productos mediante refinamiento y fue apoyado por el estado que otorgo subvenciones para investigación y construcción de infraestructuras. 
La tecnología se desarrolló y fue creando nuevas dependencias, en relación al uso del automóvil, el teléfono, la radio, el desarrollo de los plásticos, la televisión, el avión y las computadoras. El ser humano se robotizó y la cultura y la naturaleza fueron perdiendo sentido. Estados Unidos ya no era autosuficiente desde el punto de vista energético y necesito petróleo del medio oriente por lo que extendió su domino en esa zona mediante su poder militar. Con el surgimiento de las trasnacionales Estados Unidos controlaba sectores enteros comerciales a nivel internacional.

La crisis energética ha sido determinante para la quiebra del keynesianismo ${ }^{1}$ y neoliberalismo ${ }^{2}$. El petróleo sube sus precios debido a dos crisis de conflicto en el medio oriente; embargo árabe y a que Estado Unidos había alcanzado su pico máximo de extracción. Se presenta la crisis económica incrementada por la baja productividad, la sobreabundancia de capital y el colapso a escala mundial de los mercados de propiedad inmobiliario. El dólar se devaluó. Estados Unidos perdió poder económico, sin embargo, a través de políticas neoliberales mantuvo su poder hegemónico provocando la guerra en las áreas donde había petróleo. Se instala la economía global. Se crean grandes contrastes con ciudades globales y ciudades en miseria.

La expansión del capitalismo basado en energía fósil ha destruido las culturas populares clasificándolos como retrasada, valorando el conocimiento global como superior a lo local como lo menciona Sousa Santos (2010), dentro de la sociología de las ausencias como "aquellos conceptos que son desterrados como no válidos por la cultura dominante; Los saberes tradicionales frente a la ciencia, la diversidad cultural como momento del pasado en la línea ascendente del

1 Es una teoría económica propuesta por John Maynard Keynes, basada en el análisis de las causas y consecuencias de las variaciones de la demanda agregada y sus relaciones con el nivel de empleo y de ingresos. 2 Es la corriente económica y política capitalista, que apoya una amplia liberalización de la economía, el libre comercio en general y una drástica reducción del gasto público y de la intervención del Estado en la economía en favor del sector privado, que pasaría a desempeñar las competencias tradicionalmente asumidas por el Estado. progreso, la distribución de los privilegios en base a la etnia y el sexo, la valoración de la naturaleza y del trabajo humano solo si son económicamente productivos".

El modelo de crecimiento está en crisis, ya no se puede crecer infinitamente con recursos finitos. Ante tal situación de caos, deterioro, contaminación, desforestación, pérdida de la biodiversidad, desigualdades, fantasías, es que se deben presentar nuevas alternativas para el desarrollo de los seres humanos. El planeta desbordó su biocapacidad. Se debe crear consciencia de la realidad de lo que se tiene en el presente, de los límites, para hacer propuesta para el mismo presente y para el mañana. El Informe sobre los Límites del crecimiento (1972), mejor conocido como Informe Meadows ${ }^{3}$, ponía en reflexión al mundo de una grave crisis medioambiental.

\section{Por un modelo de cambio, reintegración y de desarrollo conjunto}

Han surgido nuevos paradigmas, algunos siempre basados en la parte económica, otros como disfraces del modelo actual y otros que deben analizarse para guiar junto a la realidad cultural, social y ambiental en la toma de decisiones de lo que cada pueblo quiere alcanzar. En la primera conferencia internacional de la ONU en Estocolmo 1972, sobre la problemática ambiental, se establece que la lucha contra la pobreza es esencial para proteger el medio ambiente resaltando los problemas de erosión, desertificación, gestión de bosques tropicales y contaminación de los recursos hídricos.

3 Presentado por Dennis Meadows en 1972, y en el que por primera vez en la historia de la humanidad se plasma la grave crisis ecológica que afecta al planeta, un hecho sin precedentes, ya que es creado por el propio ser humano y pone en riesgo una gran parte de la vida en la tierra. Sus previsiones a 50 años fueron tratadas de alarmistas, pero, unos 40 años después, muchas de sus conclusiones han ido cumpliéndose, por lo que se hace extremadamente importante sentar las bases de un cambio en la gestión de los recursos. Gracias a este informe, la conciencia social despertó. 
En el informe Brundtland (1987), se impulsa el concepto de desarrollo sostenible. Para Fernández y González (2014) esta concepción "vinculaba directamente el deterioro ambiental a la pobreza, al tiempo que se resaltaba que el desarrollo (crecimiento) en el Centro estaba permitiendo resolver los problemas ambientales". Para otros es un término ambiguo como señala Murray (2012), "el sustantivo, "desarrollo" (o, mejor dicho, crecimiento), se imponía claramente sobre el adjetivo, "sostenible". Sin embargo, el término era lo suficientemente ambiguo como para contentar a todo el mundo".

En la cumbre de la tierra (1992), se asigna al término desarrollo sostenible, la manera de terminar con la pobreza y resolver la problemática ambiental. Esto fue considerado por Fernández y González (2014) como un disfraz para hacer creer al mundo que ya se encaminaba hacia la sostenibilidad. Ese mensaje perduró hasta los primeros años del nuevo milenio; mientras los capitales seguían fluyendo en las élites de la aldea global.

Jiménez Herrero (2000), liga el concepto a una forma de vida, "el desarrollo sostenible se puede vislumbrar ahora como un conjunto de relaciones entre sistemas (naturales y sociales), dinámica de procesos (energía, materia e información) y escalas de valores (ideas y ética)" (pág. 109). Este sistema debe soportar los cambios y mantener la unidad de funcionamiento para que sea estable.

De tal manera, que se abordará el desarrollo sostenible más allá de las versiones oficiales, desde la construcción consciente que implica un cambio que permita integrar al ser humano a la biosfera. Las fuentes teóricas son varias; la teoría de la complejidad, la teoría de sistemas, la filosofía intercultural, el pensamiento ecológico que proviene de diversos tejidos sociales y la conciencia personal. Jabareen (2008), identifica siete conceptos que juntos sintetizan y establecen una propuesta de marco conceptual, como son: "paradoja ética, capital natural, equidad, ecoforma, gestión integral, utopía y agenda política global"

Un modelo macro del medio ambiente y de impacto humano fue propuesto por Herlich y Holdren (2014), llamado ecuación IPAT y que, "permite evaluar el impacto ambiental I, a partir del producto de tres variables: la población $\mathrm{P}$, que produce ese impacto, el consumo $\mathrm{C}$ de bienes y servicios que ella realiza para satisfacer sus necesidades $\mathrm{y}$, el impacto ambiental $\mathrm{T}$ producido por la tecnología disponible para producir esos bienes y servicios. Este modelo no es para hacer cálculos absolutos sino para tener en cuenta los pesos comparativos de cada una de las variables. De esta manera se toma en cuenta el crecimiento poblacional, el consumo y la eficiencia de la tecnología.

En este caso la ecuación IPAT, se analiza con el fin de establecer las dimensiones de las múltiples variables que inciden. En ese sentido la variable población determina el análisis que se debe realizar desde la dimensión demográfica. El consumo de bienes es una variable que se puede realizar desde la dimensión personal y comunitaria, la variable tecnología aporta a la dimensión técnica y social y la variable I se enmarca en la dimensión ambiental como resultado de la interrelación de las otras variables. A cada dimensión se le relacionará con principios o verdades fundamentales relacionadas a sostenibilidad con el fin de dar fundamento al enfoque.

En la dimensión ambiental es importante establecer el principio de conservación del capital natural. Este es el mejor indicador de la riqueza de un país. El capital natural aporta las condiciones que hacen posible la vida y es la base de la producción aportando bienes y servicios ecosistémicos. Cada país debe cuantificar sus servicios ecosistémico, valorar, preservar y recuperar su capital natural. En Nicaragua se tiene un conocimiento incompleto de la diversidad biológica, han desaparecido amplias zonas boscosas debido a; la deforestación para la urbanización, explotación maderera, la ganadería, la frontera agrícola. Según 
Lezama López (2007) "el caso más dramático ocurrió con el bosque seco tropical y semicaducifolio, el que se redujo en 500 años a menos del 10\%" (pág. 8). Así mismo MARENA ${ }^{4}$ (2010), confirma que, "las pérdidas de los elementos de la diversidad biológica, se dieron principalmente en la ecorregión del bosque tropical seco del Pacífico del país, donde las actividades agropecuarias y las malas prácticas han sido las principales causas de la degradación de los ecosistemas". En el capital humano de Nicaragua se debe contar que posee variadas fuentes naturales de energías como recursos geotérmicos, hídricos, eólicos, biomásicos, solar y marítimo.

La dimensión demográfica presenta una situación de urgencia del planeta, ya que la población crece según tasas que los recursos ambientales no pueden sostener, ni dar las condiciones básicas de salud, alimentación y energía. A nivel mundial en los últimos años se observan algunos cambios de tendencias demográficas, donde contrastan el escaso crecimiento y envejecimiento poblacional en los países desarrollados, en contraste con el dinamismo demográfico de la región asiática. Según datos del fondo de población de las naciones unidas UNFPA, Nicaragua cuenta con una población de $5,962,782$ habitantes, que creció aceleradamente entre 1950 y 2015 en un 4,2 mientras que desde el 2005 hasta el 2050 crecerá en un 1,4 veces. El descenso en el ritmo decrecimiento se debe a dos causas por un lado a que las mujeres tienen hoy día menos hijos, bajando de 7 a 3 hijos por mujer, influidas por la migración del campo a la ciudad, la educación y el uso de anticonceptivo y el otro aspecto es la migración de nicaragüenses a Costa Rica, Estados Unidos, El Salvador y España estimada en un millón de personas que envían remesas a Nicaragua consideradas en un $10 \%$ de la riqueza producida en un año. Estas consideraciones tienen su influencia en el consumo.

4 Ministerio del ambiente y los recursos naturales en Nicaragua. Institución encargada de la conservación, protección y el uso sostenible de los recursos naturales y del medio ambiente.
La variable consumo se analiza desde, la dimensión personal, comunitaria y el principio de calidad de vida. El fin no es hablar del desarrollo material sino de la calidad de vida como principio de sostenibilidad relacionado al bienestar integral, que requiere de satisfacer ciertas necesidades humanas, pero considerando el impacto ambiental de los productos y servicios consumidos. El desarrollo se refiere a las personas y no a los objetos. Para los diversos tipos de organizaciones comunitarias este objetivo de calidad de vida puede lograrse de diversas maneras. Max-Neef (1998) afirma que "el mejor proceso de desarrollo será aquel que permita elevar más la calidad de vida de las personas".

Para saber a qué, se refiere el concepto de calidad de vida, el mismo Max-Neef la determina como "La calidad de vida dependerá de las posibilidades que tengan las personas de satisfacer adecuadamente sus necesidades humanas fundamentales" (1998). $\mathrm{Y}$ las necesidades las categoriza en "existenciales y axiológicas", clasificándolas, por una parte, "en las necesidades de ser, tener, hacer y estar"; y, por la otra, "las necesidades de subsistencia, protección, afecto, entendimiento, participación, ocio, creación, identidad y libertad".

Max-Neef (1998) formula los siguientes postulados que sirven para desaprender ciertas concepciones "Las necesidades humanas fundamentales son finitas, pocas y clasificables. Las necesidades humanas fundamentales son las mismas en todas las culturas y en todos los períodos históricos. Lo que cambia, a través del tiempo y de las culturas, es la manera o los medios utilizados para la satisfacción de las necesidades"; es decir los satisfactores. Sigue mencionando Max-Neef que "una sociedad sana debe plantearse, como objetivo ineludible, el desarrollo conjunto de todas las personas y de toda la persona".

Nicaragua es un país, que ha pasado de una larga historia de guerras, sufrimientos y pobrezas, pero que al alcanzar la paz se ha propuesto vivir bien y mejorar 
su calidad de vida. En ese sentido en Nicaragua se está implementando un modelo socialista, cristiano y solidario centrado en el desarrollo del ser humano y de las familias nicaragüenses, que garantice la calidad de vida del nicaragüense en educación, salud, seguridad alimentaria, respeto al medio ambiente que garantice el bien común y la equidad social de la familia nicaragüense. El modelo del GRUN (2012) "se basa en valores cristianos, ideales socialistas y prácticas solidarias que guían la construcción de círculos virtuosos de desarrollo humano con recuperación de valores, la restitución de derechos, y el fortalecimiento de capacidades que han permitido ir superando los círculos viciosos de pobreza y subdesarrollo"

La siguiente dimensión está relacionada a la variable tecnología que viene a ser el impacto ambiental del uso de la tecnología en la producción de los bienes y servicios. Sobre la tecnología actual Wolfgang Sachs ${ }^{5}$, señala; "los instrumentos técnicos modernos más que instrumentos aislados se presentan como un inmenso sistema tecno-social formado por el conjunto de sus requisitos técnicos, socioculturales y ambientales".

Con respecto al impacto ambiental de la tecnología se propone el principio de biocompatibilidad o ecoforma. La tecnología ha tenido su impacto ambiental desde la revolución industrial, sin embargo; es desde hace dos décadas que se ha prestado interés a las evidencias del impacto ambiental y se han elaborado nuevas propuestas tecnológicas menos agresivas con el medio ambiente; como la ecología industrial, la edificación sostenible, la ecología urbana, la agricultura ecológica, la química verde, la permacultura, la escala adecuada de los sistemas técnicos tanto en su dimensión espacial como temporal. Uno de los subprinicipios es la ecoeficiencia que para $\mathrm{WBCSD}^{6}$ "significa añadir cada

\footnotetext{
5 Nació en Múnich (Alemania) es investigador, escritor y profesor universitario en el ámbito del medio ambiente, el desarrollo y la globalización. Editó y fue co-autor del Diccionario del Desarrollo: Una guía del Conocimiento como Poder que ya un 'clásico' en los estudios del Post-Desarrollo y que ha sido traducido a varios idiomas.

6 El Consejo Empresarial Mundial para el Desarrollo Sostenible, también conocido por sus siglas en inglés WBCSD, es una asociación mundial de más de 200 empresas que trabajan exclusivamente con el sector empresarial y el desarrollo sostenible.
}

vez más valor a los productos y servicios, consumiendo menos materias primas, generando cada vez menos contaminación a través de procedimientos ecológica y económicamente eficientes y previniendo los riesgos".

Las variables población, consumo y tecnología de la ecuación IPAT, se analizan en conjunto, desde la dimensión global local por el impacto ambiental que tienen las diversas poblaciones. A esta dimensión se relaciona la equidad y la solidaridad inter e intrageneracional. En el modelo actual de desarrollo se está derrochando la herencia de las futuras generaciones. Es como si no cuidara, lo que le deja a su hijo y le hereda desastre y ruina. Otro principio es el de sostenibilidad global ya que no se puede pensar en sostenibilidad local a costa de importar su impacto ambiental a otros lugares. El principio de la sostenibilidad local conceptualizado por Cendra, Cruz y Stahel (2007) "tanto desde el punto de vista ambiental como social, todo lo que pueda resolverse a nivel local debe de primar frente a cualquier solución planteada a niveles superiores" (pág. A17).

Por último, la dimensión cultural abarca toda la ecuación, ya que se relaciona a todos los demás cambios. Para Ángels Canadell (2007), los ejes sobre los cuales vertebrar la sostenibilidad como visión del mundo:

Interdependencia: expresa la interconexión mutua entre todos los fenómenos, seres y dimensiones de la realidad. La materia y la conciencia; el cosmos y los seres humanos. No-dualidad: indica la estructura relacional de la realidad. No-linealidad: el tiempo no es una línea unidireccional ni tampoco una fórmula abstracta con la que podemos hacer cálculos. Relacionalidad: todo lo que existe es relativo a un contexto, a un lugar, a una cultura.

Morin, apuesta por lo que denomina la 'cultura fundamental' que se basa en los siguientes fundamentos: El conocimiento como fuente de error e ilusión, racionalidad, cientificidad. Qué es ciencia, 
cuáles sus fronteras, sus límites, sus posibilidades, sus reglas. Qué es complejidad.

Ligado a la dimensión cultural se encuentra el principio de la educación integral que resume Delors en su informe a UNESCO (1997); "aprender a conocer, aprender a hacer, aprender a vivir juntos y aprender a ser".

La cultura es pilar importante en el desarrollo sostenible por la cohesión que proporciona a las comunidades locales, pudiendo incidir en las decisiones políticas, económicas, financieras y ambientales y de esta manera vincular la cultura con el desarrollo de los pueblos. La diversidad cultural crea un mundo de capacidades, visiones y valores humanos que unidos y tomados en cuenta representan una gama de alternativas para solucionar problemas, Definitivamente la cultura representa gran potencial para contribuir a disminuir la pobreza, el hambre, las enfermedades, el analfabetismo para lograr calidad de vida, igualdades humanas y sociedades inclusivas.

\section{Ciencia Tecnología y Sociedad desde la perspectiva latinoamericana}

La UNESCO (1999), sostiene que: "Hoy, más que nunca, no puede haber desarrollo sin ciencia y sus aplicaciones". Analizar el impacto de la ciencia y la tecnología en el ámbito latinoamericano no es fácil, puesto que, en la problemática, que tienen que enfrentar las personas, los factores que inciden, son innumerables y no todo se resuelve con ciencia y tecnología. Polcuch (2000) al respecto mencionaba "Al considerar propuestas para la medición del impacto social de la ciencia y tecnología en América Latina, no debe dejarse de lado la necesidad de tener en cuenta las características particulares de las sociedades periféricas, en especial las dificultades de diversa índole de los actores sociales para apropiar el conocimiento, ya sea transferido del exterior, o producido localmente" (pág. 19).
En estos países es importante determinar la relación entre desarrollo científico y tecnológico y el nivel de desarrollo social. El quehacer de la ciencia en América Latina, está destinada a estudiar asuntos que no tienen utilidad práctica o está enfocada en estudios relacionados a la pobreza, el desempleo, las desigualdades, el conocimiento local. Se investiga para el capital o se investiga para la sociedad. Mario Bunge (1968), resume este aspecto así, "La idea más difundida de lo que debiera ser la ciencia en los países en desarrollo parece ser ésta: debiera ser empírica antes que teórica, regional antes que universal, aplicada antes que pura, natural antes que social, y en todo caso filosóficamente neutral" (pág. 4), lo que indica que el reparto no solo es de tierras y riqueza sino también de la ciencia y es terminante Bunge cuando dice, "estas cinco tesis de la filosofía popular del desarrollo científico en los países en desarrollo son nefastas: de aplicarse distorsionarían y retardarían el avance de la ciencia" (pág. 8).

En la práctica, éstas falsas filosofías del desarrollo científico se han aplicado y de ahí se deriva el atraso de la ciencia en los países latinoamericanos a pesar de que, Bunge, en el mismo escrito plantea la solución con reemplazar la filosofía fragmentada, con una filosofía integral de la investigación científica sintetizada de la siguiente manera "Una adecuada filosofía de la investigación científica deberá reconocer que esta es una empresa multifacética: que tiene un lado teórico y otro empírico; que es universal en cuanto a su método y su finalidad, aun cuando en cada región posea objetos o temas típicos; que tiene un lado puro y otro aplicado; que se ocupa tanto de la naturaleza como del hombre; y que tiene supuestos filosóficos tanto como resultados de importancia filosófica" (pág. 7).

En Nicaragua también se apuesta por una filosofía de investigación integral, como se menciona en el $\mathrm{PNDH}^{7}$.

7 El Plan Nacional de Desarrollo Humano PNDH; persigue la prosperidad que conduce a nuestra sociedad por la senda del buen vivir, en paz, armonía, en comunidad; que a su vez promueve nuevas opciones de acción social, de consenso. El PNDH es un plan vivo, en continua construcción, abierto a los aportes de la sociedad nicaragüense, que es actualizado de manera periódica para ajustarse a los procesos cambiantes internos y externos. 
La política de Investigación, Desarrollo e Innovación $(\mathrm{I}+\mathrm{D}+\mathrm{i})$ debe ser integral y reformar todo el proceso de generación de tecnología y su aplicación en el sistema productivo (GRUN, 2012). Para alcanzar tales retos es necesario vincular a investigadores, innovadores, desarrolladores de tecnologías y usuarios de la ciencia y tecnología, ya que existen limitantes como menciona el PNDH "Nicaragua es uno de los países con más baja inversión en investigación y desarrollo (I+D) a nivel de América Latina. Mientras Brasil y Chile invierten el 1.0 y 0.7 por ciento de su PIB respectivamente; y Costa Rica, otro país centroamericano, invierte 0.32 por ciento; Nicaragua solamente invierte 0.05 por ciento" (GRUN, 2012, pág. 102).

Las PYMES trabajan con tecnología atrasada y practicas obsoletas, influyendo negativamente en la producción. El cambio climático está teniendo su efecto negativo en el sector productivo mediante sequias o inundaciones por lo que urgen mecanismos de adaptación el cambio climático como sigue mencionando el GRUN (2012), "a partir de una mayor capacidad innovadora productiva y social; generación y adaptación de tecnologías aplicadas a cada cultivo y a cada región productiva, y la generación y transferencia de buenas prácticas de producción".

La problemática de la ciencia y tecnología en América Latina se debe analizar desde la perspectiva estructural, porque no es un problema lineal de etapas, de pasar de un estado de subdesarrollo al desarrollo de manera continua, sino que es un estado en el que nos ha metido el propio sistema para garantizar el atraso. Por lo tanto, no se resuelve solamente con la parte material de asignación de más tiempo a la investigación, de creación de laboratorios, o de mejoras salariales, de evitar la fuga de cerebros, de tener asesoría técnica internacional como muchos piensan. Tiene que ver con otros asuntos que señala Osvaldo Sunkel (1969); en América Latina se han incorporado avances tecnológicos a lo largo de los años; pero no por esfuerzo investigación y adaptación a las necesidades propias, como en los países industrializados, sino por simple traslación desde otros países sin considerar suficientemente las condiciones y necesidades fundamentales del país y, por tanto, sin contribuir a ampliar su base científica y tecnológica.

Lo anterior, ha dado lugar a un considerable rezago tecnológico general y a que subsistan en América Latina graves desequilibrios internos: junto a los métodos más modernos de producción y consumo, de investigación y educación, hay en amplios sectores con formas primitivas, de escaso rendimiento, y niveles culturales y científicos bajos.

Sunkel (1969), menciona que la revolución industrial no nos fue ajena ya que Latinoamérica tuvo un papel activo como productores de las materias primas y alimentos que requerían los países del centro, así como abriendo nuestros mercados a los productos manufacturados en que ellos se especializaban, y llegando en períodos más recientes incluso a producir muchos de esos bienes. Desde este interesante enfoque se refleja que a lo largo de la historia se ha mantenido y utilizado estrecha relación de técnicas avanzadas que se han incorporado en las diversas facetas de la sociedad latinoamericana. Toda esa tecnología trasladada no ha provocado las transformaciones socioeconómicas que provocaron en los países desarrollados, ni ha aumentado la productividad, ni la eficiencia productiva.

Se produjo división del trabajo entre los países que hacen ciencia y tecnología moderna de transporte, comunicación, salud, energía y los que hacen materia prima alimentos, insumos, minerales. De igual manera se reparte la creación de tecnología en aquellos países para avanzar su desarrollo y aplicarlas en la producción, comunicación, salud y energía, mientras que los países en subdesarrollo recuren a traer tecnología cuando se presentan problemas, creándose una dependencia en ciencia y tecnología.

Como dice el mismo Sunkel (1969), la universidad no ha jugado ningún papel clave en esta transferencia ya que la tecnología ha venido por otros canales. El 
brasileño Leite López (1969) es más crudo en su análisis respecto al papel que ha jugado la universidad "Si se llegara a cerrar una de las grandes universidades de un país de América Latina, el sistema económico de ese país no sufriría ninguna alteración. (...) La economía continuaría, como ha sucedido en el pasado, dependiente de la técnica externa que el país compra o arrienda, como si fuese una fatalidad histórica" (pág. 32)

La empresa latina pasó de vender productos importados y algunos artesanales a crear un proceso de sustitución de importaciones manufactureras dando lugar al proceso de industrialización, pero con unas bases precarias de infraestructura, estructura y superestructura industrial y la ausencia casi total de la actividad científica, que al final terminó convirtiéndose en una industria de bienes y consumos. En los casos donde no ejercía influencia el mercado se siguió una política activa de oferta, de transformación deliberada de la estructura productiva como es el caso de la energía hidroeléctrica y del petróleo. La energía en vez de impulsar la industria pesada y la transformación de la producción rural se convirtió en un subsidio para estimular la utilización indiscriminada de artefactos eléctricos de consumo. El acero en vez de utilizarse para la industria de maquinarias e implementos agrícolas, fue a parar a las plantas ensambladoras de automóviles y a las construcciones residenciales.

Mientras tanto, el modelo de industrialización en los países desarrollados se ha venido estructurando en torno a grandes monopolios multinacionales, cuyos núcleos de investigación, desarrollo e innovación se encuentran en los países industrializados y desde los que crean nuevos productos, procesos, maquinaria para producirlos, materia prima sintética y publicidad para dinamizar los mercados. A los demás países se les deja la utilización. Como menciona Sunkel (1969), aparece "la misma especialización del centro en la generación del nuevo conocimiento científico y tecnológico, y de la periferia, en su consumo y utilización rutinaria”.
En algunos casos la industria de países latinoamericanos se ha desarrollado basada en la importación de tecnología, para fabricar productos inventados en otros países, utilizando patentes arrendadas. En esas industrias el ingeniero formado en las universidades no tiene posibilidades de ejercitar su capacidad de inventar o de investigar para perfeccionar las técnicas. Leite López (1969) afirma "Por consecuencia, es perfectamente claro que las dificultades del sistema educacional, la estructura inadecuada de nuestras universidades, las deficiencias crónicas existentes en nuestros institutos científicos, no son fortuitas. Ellas provienen del hecho de que, en el sistema económico, la industria, la agricultura, se basan en un sistema de trabajo, en una organización política, económica y social que no exige que nuestro sistema educacional o universitario y la investigación científica y tecnológica produzcan elementos para ser absorbidos por ese sistema económico.

Ante estas situaciones, se deben definir políticas de desarrollo que se enfoquen en economías nacionales autónomas y dinámicas que impulsen la investigación que produzca impacto en las problemáticas nacionales, territoriales y locales, como señalan Sábato y Botana (1968), "La superación del desarrollo de América Latina resultará de la acción simultánea de diferentes políticas y estrategias. En todo caso, y cualesquiera sean los caminos elegidos, el acceso a una sociedad moderna-que es uno de los objetivos que se pretenden alcanzar por el desarrollo- supone necesariamente una acción decisiva en el campo de la investigación científico-tecnológica" (pág. 2).

Estos autores, consideran que, la investigación científico-tecnológica es una poderosa herramienta de transformación de una sociedad y por lo tanto la estrategia de Latino América para alcanzar desarrollo social, económico, político y cultural Por lo que se debe impulsar la investigación científica tecnológica, "América Latina, deberá cambiar su papel pasivo de espectador por el activo de protagonista, procurando conquistar la máxima participación". 
En relación a los aportes científicos técnicos vinculados al desarrollo autónomo Osvaldo Sunkel (1969) menciona:

la posibilidad del desarrollo autónomo se refiere a transformar el sistema productivo interno en una estructura flexible, dinámica y capaz de generar un apreciable excedente propio de recursos de inversión; vinculaciones externas que conlleven a relaciones internacionales tendiente a desmontar y desarticular el mecanismo de la dependencia inherente en el modelo centro-periferia, y la creación de una infraestructura científico-tecnológica capaz de apreciar críticamente y de transferir y adaptar selectivamente el progreso científico-tecnológico generado en cualquier parte del mundo, así como de generar nuevos aportes propios a la ciencia y la técnica. La autonomía se podrá alcanzar cuando además de usar tecnología ya descubiertas y comprobadas, se utilicen nuevas tecnologías creadas por la masa gris nacional para resolver los problemas propios, basándose en la innovación más que en la imitación (pág. 81).

La investigación en la universidad debe estar estrechamente vinculada a la producción, al entorno, a la solución de problemas socioeconómicos y ambientales. El profesional debe estar capacitado para aplicar lo que aprendieron en las universidades, fortaleciendo capacidad de inventar, de crear, sus cualidades de investigación para perfeccionar técnicas, proceso y productos.

Sunkel (1969) manifiesta "para crear una nueva estructura productiva interna, se requiere un parque científico y tecnológico apropiado para nuestras necesidades, la capacidad de exportación de productos manufacturados, la capacidad de penetración dinámica en una gran variedad de mercados externos, la capacidad de resistencia ante los efectos de las sociedades avanzadas de consumo" (pág. 81). Sábato y Botana (1968), refiriéndose a la infraestructura científico tecnológico, sugieren los siguientes elementos:

El sistema educativo, que produce en la calidad y cantidad necesaria los hombres que protagonizan la investigación: científicos, tecnólogos, ayudantes, asistentes, operarios, administradores; los laboratorios, institutos, centros, plantas piloto donde se hace investigación; el sistema institucional de planificación, promoción, coordinación y de estímulo a la investigación, los mecanismos jurídico-administrativos que reglan el funcionamiento de las instituciones y actividades y los recursos económicos financieros aplicados a su funcionamiento (pág. 3).

En cuanto a la innovación es necesario construir el camino, orientándolo hacia un nuevo enfoque que funcione en Latinoamérica totalmente diferente a como se ha manejado hasta ahora, iniciando por cambiar a las personas, antes de usar tecnología, para cambiar a las personas. La innovación moviliza la imaginación, capacidad y compromiso de los que desean construir "algo diferente", o "hacer de forma distinta" lo que se hace de forma ordinaria y hasta una nueva forma de interpretar la realidad como enfatizan José de Souza Silva (2004) y otros autores "Todos somos intérpretes de la realidad. Sin embargo, no todos realizamos interpretaciones a partir de marcos interpretativos autóctonos que, desde las historias y aspiraciones locales, realicen un esfuerzo interpretativo independiente del pensamiento subordinado al conocimiento autorizado por el más fuerte".

La innovación autóctona tiene que considerarse desde los territorios con sus recursos humanos, naturales, económicos, institucionales, culturales que son el potencial del desarrollo endógeno. Brunet y Baltar (2010) enfatizan en el factor clave que beneficie a las fuerzas locales "existencia de un sistema regional de innovación o aprendizaje, basado en mecanismos sociales y relacionales, y apoyado en instituciones adaptadas a las características y necesidades del sistema productivo local" (pág. 14). Sin embargo, hay que considerar las dinámicas locales y los distintos niveles de gobierno. Para Ricardo Méndez (2002) las condiciones para la innovación están dadas por "la presencia de un sistema productivo vinculado a una o varias actividades, en el que buena parte de las empresas existentes realizan esfuerzos en el plano de la innovación tecnológica, incorporando mejoras en 
los diferentes procesos asociados a su cadena de valor y en los productos (bienes materiales/servicios) que ofrecen” (pág. 9).

En Nicaragua, en los territorios se implementa un modelo sistémico de innovación tecnológica. Este modelo según Pedroza (2010), debe ser "integral e integrador, con énfasis en los enfoques, sistémico, IESA $^{8}$, participativo, competitivo, cadenas productivas del agro-negocio, guiado por la demanda tecnológica de los productores, conservacionista, colaborativo, multidisciplinario, e interinstitucional, que contribuya por esta vía a erradicar la pobreza rural y urbana en el país. Este modelo está desarrollando capacidades locales en los territorios que promueven el aprendizaje local y socializa el conocimiento" (pág. 81). En Nicaragua se están desarrollando acciones innovadoras en el área de energías renovables como el uso dela biomasa en sistemas de cogeneración en los ingenios, eco fogones, mini hídricas, biogás, eólicos y solares fotovoltaico para iluminación y bombeo.

\section{Contextos energéticos actuales}

En el contexto energético mundial según Álvarez y Suárez (2016), se han observado algunos cambios como la independencia energética exterior de Estados Unidos basada en el uso de tecnologías carísimas y contaminante como la perforación horizontal, fracking o fractura hidráulica, para obtener gas de esquisto o shale y petróleo fracking lo que ha provocado brusca caídas del precio del petróleo y gas debido a aumento en la oferta, baja en la demanda y a aspectos geopolíticos y devuelto el poder energético a Estados Unidos para hacer influencia en precios mercados y aspectos políticos. Otra vez para garantizar la seguridad y hegemonía energética de los países desarrollados no importa el daño ambiental que provocan sobre todo en los cuerpos de agua ya que utiliza químicos extremadamente contaminantes para

8 Enfoque integrado de investigación y extensión en sistemas agropecuarios. Es una vía para la adopción y aplicación de nuevas tecnologías, como clave de la innovación tecnológica. que el agua mantenga la presión en las largas tuberías. Esto afecta a los productores tradicionales de petróleo cuyos países verán disminuidas sus inversiones.

Otra característica importante actual es la disminución considerable del consumo de energía en China. Basado en datos de la agencia internacional de energía, Pascual (2016), señala, el crecimiento en la mayoría de los sectores de gran consumo energético de China, como el acero, el hierro y el cemento disminuyeron apreciablemente en 2014 en relación al aumento intensivo que tenía en los últimos años. Este patrón cambiante de crecimiento económico hizo que el crecimiento del consumo de energía de China aumentase un 2,6\% en 2014, lo que representa menos de la mitad de su promedio en los últimos 10 años $(6,6 \%)$ y la tasa más débil de crecimiento desde finales de los 90 . Esto hace considerar una caída adicional en la demanda.

La India acelera su modernización y su base industrial, está entrando en un periodo de crecimiento de consumo energético, aumentando la demanda de carbón en la generación eléctrica e industrial. Hay que recordar que La India es uno de los países con menos acceso a la energía eléctrica, se estima que unos 7,5 millones de familia no tienen acceso a la energía eléctrica. El empuje energético es de interés y de vigilancia por el enorme compromiso de capital, las tecnologías a usar, el impacto ambiental que se puede provocar y el consumo de bienes y servicios.

En general la demanda mundial, sigue creciendo, el consumo en el año 2015 de energía primaria creció el $1,0 \%$, similar a la tasa de crecimiento vista en 2014, pero mucho más lento que el promedio observado en la última década. Basados en análisis de la agencia internacional de energía las tendencias hacia el futuro de la energía a nivel mundial son: Los hidrocarburos, junto con el carbón, llevan camino de seguir dominando la combinación de fuentes de energía primarias del mundo, al menos hasta el año $2030 \mathrm{y}$, posiblemente, hasta bastante más tarde. Las necesidades mundiales 
totales de energía son un 55\% mayor en 2030 que actualmente.

En Nicaragua según $\operatorname{INE}^{9}$ (2016), durante el año 2015 la economía nicaragüense continuó fortaleciéndose, creciendo el PIB en 4.0 por ciento y la inflación fue del 3.5 por ciento. Así mismo, desde el año 2007 Nicaragua ha venido transformando su matriz energética. De producir solo el 25 por ciento de su energía con fuentes renovables, a 52 por ciento en el 2016 y con una meta para 2018 de 74 por ciento.

Nicaragua impulsa su transformación energética, en momentos en que el petróleo alcanzaba niveles altos de costo. Según INE (2016), a mediados del 2014 el precio del petróleo comenzó a caer y el país pagaba el 50.7 más barato. El usuario debería pagar menos pero aún no está sucediendo ya que paga 0.21 dólares por kilovatio hora, debido a que Nicaragua tiene una deuda que pagar y una estructura de subsidios cruzados con más de 80 por ciento de los usuarios. El pliego tarifario debe revisarse, ya que fue diseñado hace más de 30 años cuando el país pasaba por otra realidad económica y eléctrica diferente. Según estimaciones de Zamora ${ }^{10}$, Nicaragua tiene 21 por ciento de pérdidas en la distribución de energía, cuando el promedio en la región es del 11 por ciento.

Según la empresa nacional de transmisión eléctrica y el ministerio de energía y minas- ENATREL Y MEM (2016)de Nicaragua, "en el 2016 alcanzaremos uníndice de cobertura eléctrica nacional del $90 \%$, electrificando 86,553 viviendas urbanas y rurales, atendiendo más de 452,000 protagonistas con la construcción de 623 Kilómetros de redes de distribución de energía eléctrica". Según el plan de buen gobierno 2016 "se estima que la generación renovable será del $52.5 \%$ y el $47.5 \%$ en base a petróleo". Consultada la base de datos de ENATREL el día 20 de noviembre del presente año, "la generación se compone de un $69 \%$ basada en energías renovables y $31 \%$ en no renovable basada

9 Instituto Nicaragüense de Energía

10 César Zamora, presidente de la Cámara de Energía de Nicaragua en bunker. La renovable se constituía en un $30 \%$ de eólica, $17 \%$ de geotérmica, $11 \%$ de hidroeléctrica y $11 \%$ basada en biomasa"

Para el desarrollo del sector energético, los países de la región cuentan con un portafolio de posibilidades CEPAL SICA (2007), entre las cuales se puede mencionar el uso racional y el incremento de la eficiencia energética (la única que se puede ejecutar al corto plazo, con efectos inmediatos), recursos hidroeléctricos y geotérmicos, energía eólica y solar, cogeneración, biocombustibles, biomasa, fuentes renovables para sistemas aislados, y combustibles fósiles (petróleo, gas natural y carbón).

Según el Ministerio de Energía y Minas. MEM (2010), el potencial eólico de Nicaragua es 800 MW pero solamente se ha instalado $78 \mathrm{MW}$. El potencial es amplio, pero aún se imponen algunos obstáculos como altos costos de inversión inicial, costos elevados de generación de energía eléctrica, bajas eficiencias en comparación con sistemas tradicionales, baja densidad energética de las fuentes, intermitencia en el recurso energético, falta de mayores políticas energéticas, marco regulatorio insuficiente y poco conocimiento de las tecnologías existentes.

Una de las estrategias sustentable centroamericana es impulsar las energías renovables de generación distribuida como se menciona en el informe del CEPAL SICA (2007), "las fuentes de energía renovable, consideradas a pequeña escala, tienen el enorme potencial de proporcionar energía a poblaciones que se encuentran en zonas aisladas, para aplicaciones de uso domiciliar, comunitario y productivo" (pág. 63). Además, es importante considerar la combinación de diversas tecnologías de tal manera que sea posible aprovechar las ventajas de las diferentes fuentes, tanto fósiles como renovables, para intentar mejorar la eficiencia energética total.

Los sistemas eólicos distribuidos pueden utilizarse para abastecer comunidades de poca población y 
según Caravaca, González, y Silva (2005) contribuyen al desarrollo territorial. En el caso del municipio de San Nicolás del departamento de Estelí, Nicaragua, partiendo de evaluar y aprovechar el recurso viento de la zona para la generación de energía eléctrica mediante tecnologías renovables eólicas. Reconociendo el rol de integrar redes que incluyen la universidad y los otros actores presentes y no presentes en la zona y el aspecto de las dinámicas de aprendizaje presente en la propuesta mediante la creación de una cooperativa eléctrica para contribuir al desarrollo socio económico del territorio.

En el aspecto técnico, según Jordi Segalás (2007), la evaluación de las turbinas eólicas debe tener en cuenta el factor de disponibilidad de vientos, la distribución de Weibull y rosa de vientos. Generalmente la potencia eólica aprovechable se expresa mediante la siguiente expresión: $\mathrm{Pd}=1 / 2 \rho A v 3$ donde $\rho$ es la densidad del aire, $\mathrm{A}$ área perpendicular al viento $\mathrm{y} \mathrm{v}$ media de la velocidad y por el teorema de Betz la máxima potencia aprovechable que se puede obtener en teoría de una corriente de aire con un aerogenerador ideal nunca puede superar al $59,3 \%$ de la potencia de viento incidente Pamax=0,593 Pd.

Según Forero Núñez, Valencia, y Sierra Vargas (2016), "El análisis y modelamiento de sistemas energéticos eólicos, también se puede hacer utilizando aplicaciones computacionales que incluyen las ecuaciones fundamentales de las tecnologías para el diseño, ecuaciones financieras para el análisis de los costos de operación, mantenimiento, análisis del impacto ambiental. Estos programas HOMER, HYBRID2, RAPSIM, TRNSYS, y RETScreen" (pág. 5).

Desde un enfoque integral es importante involucrar en la toma de decisión sobre la instalación de sistemas eólicos la percepción de la gente sobre las mejoras en las condiciones de vida, el aprovechamiento de los recursos sol y viento, la transferencia de tecnología y el posible impacto ambiental, como menciona Solorzano Morales (2017), "Las áreas geográficas aún no electrificadas, así como, las proyecciones de demanda futura de estas mismas zonas requieren de una ingeniería social que considere tanto las opciones técnicas como las características económicas, sociales y culturales de estos grupos vulnerables" (pág. 18).

\section{CONCLUSIONES}

El modelo creado desde la revolución industrial promovió el desarrollo basado en una dualidad hombre y todo lo demás, buscando por todos los ámbitos el crecimiento en dinero de las personas. No se consideró la finitud de los recursos naturales explotándose sin medidas, ni la equidad en el desarrollo, ni la diversidad cultural. Generó grandes desequilibrios en los ecosistemas, provocando el cambio climático. Creo grandes desigualdades humanas acumulando grandes capitales en una minoría y empobreciendo a la gran mayoría. Este modelo persiste hasta la actualidad, salvo en algunas naciones que han decidido por alternativas menos dañina.

El uso de la energía ha sido decisivo en la implantación de este modelo de derroche en el que se ha creado dependencia de combustibles fósiles para generarbienes y servicios de consumo para garantizar la extensión del capitalismo. El afán de dominar geopolíticamente los territorios de los yacimientos de carbón, petróleo y gas natural ha provocado guerras, manipulaciones, invasiones, segregaciones, migraciones.

Un nuevo paradigma de desarrollo ha sido introducido. El paradigma de desarrollo sostenible con sus teorías, dimensiones y principios; sin embargo, no ha sido tomado en serio por los llamados países desarrollados que lo aplican a su conveniencia o sencillamente imponiendo su poder, no se someten a los principios de desarrollo sostenible.

El desarrollo sostenible es posible, para lograrlo, primeramente, es necesario un cambio en la mentalidad de las personas porque se enecesitan desaprender ciertas concepciones y hábitos erróneos que hemos adquiridos 
del sistema olvidándonos de que la riqueza está en la relación con la naturaleza, la familia, los amigos, las culturas. La sociedad humana necesita transformarse para construir un mundo mejor y un futuro viable. El desarrollo sostenible no es lineal o triangular, sino que está estrechamente vinculado a la interrelación de varios actores en el tejido social como instituciones, gobierno, universidad, decisores territoriales, locales, la empresa, cooperativas, movimientos toda la sociedad. La autonomía energética es viable en tanto se aprovechen los recursos energéticos de manera racional y se opte por alternativas renovables.

La ciencia y la tecnología se han desarrollado para bien y para mal. Para bien por la existencia de avances que han facilitado la vida al humano en la medicina, comunicaciones, la salud y otras facetas dela vida y para mal porque los avances no han sido equitativos para todos. La ciencia no ha escapado al reparto entre los que investigan para el desarrollo de las nuevas tecnologías y productos y los que no investigan por que han sido relegados a laborar para suministrar materias primas para los productos, construcción de máquinas y nuevas tecnologías quedando sin capacidades para la investigación e innovación. La tecnología energética ha evolucionado en los países desarrollados y ahí se propagado hacia Latinoamérica como usuarios.

Las estrategias para hacer ciencia y tecnología en Latinoamérica deben enfocarse en alianza con las universidades como formadores, los empresarios apoyando económicamente y el gobierno estableciendo políticas públicas y un marco regulatorio que facilite la investigación, la innovación y el desarrollo de tecnología propia. En el área de energía renovable fomentar la eficiencia energética y el reemplazo paulatino por energías renovables.

Nicaragua definitivamente va por buen camino. Implementa un modelo socialista, cristiano y solidario que integra los factores del desarrollo humano. Impulsa la investigación y la innovación. En el aspecto energético existe un programa para la transformación de la red de energía y reducir al máximo la dependencia de combustible fósil.

La implementación de energías renovables en Nicaragua, ha crecido en los últimos años, aún con la baja del precio del petróleo, pero se requiere revisar el marco regulatorio que facilite por ejemplo la conexión de sistemas energéticos renovables a la red.

Una estrategia sustentable es impulsar las energías a pequeña escala como el uso de sistemas fotovoltaicos y eólicos para zonas rurales remotas y zonas urbanas de escasos recursos. La propuesta de sistemas de generación distribuida garantiza energía por 24 horas al día y reduce la dependencia de la distribuidora convencional.

\section{REFERENCIAS BIBLIOGRAFICAS}

Fundación Bariloche y La Universidad de Sao Paulo. (2013). Energía: Una visión sobre los retos y oportunidades en América Latina y El caribe. Sao Paulo: Corporación Andina de Fomento.

(CNDC)., E. -C. (20 de Noviembre de 2016). Generación por Tipo de Fuente. Recuperado el 12 de mayo de 2017, de http://www.mem.gob.ni

Arocena, R. (2003). Problemas del desarrollo en América Latina. Buenos Aires: Universidad Nacional de Quilmes.

Brunet Icart, I., \& Fabiola, B. (Octubre de 2010). Desarrollo endógeno, calidad institucional e innovación. Una revisión de la teoría y de algunos de sus límites. Reforma y Democracia. No. 48(48).

Bunge, M. (1968). Filosofía d ela investigación cientifica en los países en desarrollo . Acta cientifica venezolana, 19.

Caravaca, I., González, G., \& Silva, R. (2005). Innovación, redes, recursos patrimoniales y desarrollo territorial. EURE. Revista latinoamericana de estudios territoriales, XXXI(094), 5-24.

CEPAL SICA. (2007). Estrategia energética sustentable centroamericana 2020. Panamá. 
ENATREL. (2016). Octubre Victorioso: $90 \%$ de Nicaragua con energía eléctrica de calidad. Recuperado el 25 de febrero de 2017, de ENATREL. Empresa Nacional de Transmisión Eléctrica: http:// www.enatrel.gob.ni

Energía, I. N. (2016). Informe de Gestión del Instituto Nicaragüense de Energía Ante la Honorable Asamblea Nacional de Nicaragua Período 01 de Enero al 31 de Diciembre 2015. Managua.

Forero Núñez, C. A., Valencia, J. A., \& Sierra Vargas, F. E. (enero-abril de 2016). Revisión de las estrategias de modelamiento y análisis aplicados a sistemas híbridos de energía. Ingeniería Mecánica, 19(1), 59-67.

García Arostegui, D., \& Tejado Montero, L. (2012). Contracultura y ciberactivismo. Derrumbando los mitos del fetichismo digital (2/2). Nodo 50.

GRUN, G. d. (2012). Plan nacional de desarollo humano 2012-2016. Managua.

INE. (2016). Informe de Gestión del Instituto Nicaragüense de Energía Ante la Honorable Asamblea Nacional de Nicaragua Período 01 de Enero al 31 de Diciembre 2015. Managua Nicaragua: INE.

Jabareen, Y. (2008). A new conceptual framework for sustainable development, Environment, Development and Sustainability. Obtenido de y. http://www.springerlink.com/content/ v53615166x446wnh/fulltext.pdf.

Jaume Cendra Garreta, A. W. (2006). Hacia una construcción social del desarrollo sostenible basada en la definición de sus dimensiones y principios, articulados a partir de la ecuación IPAT. Aproximación a sus implicaciones y debates. Revista inernacional de ssotenibilidad tecnología $y$ humanismo(1).

Jaume Cendra Garreta, I. C. (2007). Introducción al desarrollo sostenible . Catalunia: Catedra UNESCO de sostenibilidad.

Leite Lopes, J. (Marzo/abril de 1969). Ciencia, Universidad e Realidade Nacional. Cadernos Brasileiros(52).

López, M. L. (2007). El Índice de Capital Natural como instrumento de análisis de pérdida de biodiversidad en Nicaragua. Managua.

Marcel Cano, J. C. (2004). Oikonomía vs crematística: bases de las contradicciones del desarrollo moderno. Sostenible.

MARENA. (2010). IV informe nacional al convenio sobre la diversidad biológica. Managua.

Max-Neef, M. A. (1998). Desarrollo a escala humana. Barcelona: ICARIA editorial S.A.

MEM. Dirección General de Electricidad y Recursos Renovables. (2010). Guía del inversionista. Invirteindo en el sector eléctrico de Nicaragua. Managua. Nicaragua: DGERR. MEM.

Méndez, R. (2002). Innovación y desarrollo territorial: algunos debates teóricos recientes. Scielo. EURE (Santiago) Disponible en: $<$ http://www.scielo.cl/scielo.php? script $=$ sci arttext\&pid=S0250-71612002008, 28(84), 63-83. doi:http://dx.doi.org/10.4067/S025071612002008400004

Morin, E. (2000). Los siete saberes necesarios para la educaicón del futuro. Paris: Santillana.

Pascual, J. P. (17 de Noviembre de 2016). Las tres crisis. Recuperado el 10 de mayo de 2017, de https://lastrescrisis.blogspot.com.es/2015/09/ situacion-energetica-global-2014.html

Pedroza Pacheco, M. E. (2010). Un Nuevo Modelo de Innovación Tecnológicapara el Agro Nicaragüense. Managua, Nicaragua: Editarte.

Polcuch, E. F. (2000). La medición del impacto social de la ciencia y tecnología. Buenos Aires: Universidad Nacional de Quilmes .

Prat, À. C. (2007). EDUCACIÓN SOSTENIBLE. Criterios para la introducción de la sostenibilidad en los procesos educativos. Catalunya: Cátedra UNESCO de Sostenibilidad. Universidad Politécnica de Catalunya.

Ramón Fernández Durán, L. G. (2014). En la espiral de la energía. Historia de la humanidad desde el papel de la eneergía (pero no solo) (Vol. 1). Madrid: Libros en acción.

Ritter Ortiz, W. (marzo de 2017). Ciencia, prediccción $\mathrm{y}$ entendimiento en el mundo moderno de la 
complejidad. (rcci.net, Ed.) Revista globalización. Recuperado el Noviembre de 2017

Sabato, J. A. (2011). El Pensamiento latinoamericano en la problemática ciencia - tecnología desarrollo - dependencia. Buenos Aires: EDiciones bibkioteca nacional.

Sábato, J., \& Botana, N. (1968). La ciencia y la tecnología en el desarrollo futuro de América latina. Revista de la integración.

Segalás Coral, J., López López, A., Horta Bernus, R., DiPrátula , H., \& Gásquez Rutes, X. (2007). Instalaciones aisladas y grandes parqeus eólicos. En C. U. Catalunya, Módulo 6. Energía eólica. Master en energía para el desarrollo sostenible. Catalunya: FUNDACIO UPC.

Silva, J. d. (2004). La innovacion de la innovación institucional. De lo universal, mecánico y neutral a lo contextual, interactivoy ético. Quito: Red nuevo paradigma para la innovación institucional de America Latina.

Solorzano Morales, M. A. (2017). Electrificación: La integración territorial e inclusión social en Centro América.

Sousa Santos, B. (2010). Descolonizar el saber, reinventar el poder. Montevideo: Trilce.

Suárez Diez, C., \& Álvarez Pelegry, E. (2016). Gas no convencional: shale gas Aspectos estratégicos, técnicos, medioambientales y regulatorios. Madrid: ORKESTRA. INSTITUTO VASCO DE COMPETITIVIDAD. MARCIAL PONS.

Sunkel, O. (1969). La Universidad Latinoamericana ante el a vance cientifico y tecnico; algunas reflexiones. Santiago de Chile.

UPC, F. (2007). Energía y sostenibilidad. Catalunia: Cátedra Unesco. 